Case Study

\title{
ALL MEMBERS OF A SINGLE FAMILY INCLUDING A 5-MONTHS OLD INFANT HAVING TUBERCULOSIS- A CASE REPORT
}

\author{
Chowdhury EUA ${ }^{1}$, Huq MN$^{2}$, Parveen $\mathrm{F}^{3}$ \\ ${ }^{1}$ Registrar; Department of Paediatrics, Bangladesh Medical College Hospital \\ 2Professor \& Head; Department of Paediatrics, Bangladesh Medical College, Hospital \\ ${ }^{3}$ Internee Doctor; Department of Paediatrics, Bangladesh Medical College Hospital
}

\begin{abstract}
Introduction : The incidence and number of tuberculosis (TB) cases have been increased dramatically all over the world. It is a major public health problem in Bangladesh too.

Case Summary : A 5-months old male infant, immunized as per EPI schedule, 3rd issue of a nonconsanguinous parents residence at downtown, Dhaka from a poor socioeconomic background was admitted into the Paediatrics ward, Bangladesh Medical College Hospital on 11th March 2004 with fever for 1 month, dry cough for 21 days, loose motion and vomiting for 14 days. He was weaned from breast milk at 3 months of his age and there was gross malfeeding history. BCG vaccine was given at 6 weeks. His mother had also been suffering from recurrent febrile illnesses and weight loss for many months. Baby was looking ill but conscious, mildly pale, afebrile with no dyspnoea. Z score of wt/age, Ith/age, wt/th and OFC were $-2.9,-3.5,-0.3$ and -0.3 respectively. Fontanel was open and normal. There was no lymphadenopathy and BCG mark was present. Breath sound was vesicular with fine crepitation on right lung. He was treated initially as septicemia by combined parenteral antibiotics. But response was not satisfactory. Lab data yielded raised ESR, eosinophillia, pyuria and haematuria with sterile culture. Chest $X$ ray was abnormal. Mantoux Test (MT) was found strongly positive ( $18 \mathrm{~mm} / 72 \mathrm{hrs}$.). Other family members were immediately screened for TB and all revealed positive. Complete Blood Count, MT and Chest $X$ ray were chosen as screening methods for them. We treated with anti tubercular therapy to all of them including baby. We also corrected the feeding practice of the patient and kept in close monitoring. He showed remarkable clinical improvement with weight gaining. We discharged him as disseminated tuberculosis with having pulmonary tuberculosis among all other family members and advised for follow up. They all were cured. At present, they are healthy and baby is now 4 years of age with good physical and mental growth.
\end{abstract}

Conclusion : TB can mimic everything. A high index of suspicion by the clinician is essential for early diagnosis. Message: Family members of affected child must be screened for active TB

Keywords : Tuberculosis, Tuberculosis in infant, Pan family tuberculosis.

\section{BACKGROUND}

The incidence and number of tuberculosis (TB) cases have been increased dramatically. More than 8 million of new cases of TB occur and 3 million people die /year worldwide (WHO). Almost 1.3 million cases and 450,000 deaths occur in children per year. ${ }^{1,2}$ It usually occurs in underdeveloped countries due to poverty, limitation in health-care services

\section{Correspondence to}

Dr. Ehsaan - UI - Alam Chowdhury

E-mail: ehsaanchowdhury@yahoo.com to high-risk populations. But in developed countries it is due to immigration from the prevalence countries and the epidemic of human immuno deficiency virus (HIV) infection. According to the WHO, developing countries including India, China, Pakistan, Philippines, Thailand, Indonesia, Bangladesh, and the Democratic Republic of Congo account for nearly $75 \%$ of all cases of TB. ${ }^{3}$ TB is a major public health problem in Bangladesh. Over 300,000 people develop the disease every year of whom 70,000 die. ${ }^{4}$

TB equally affects both sexes and mortality exists at the extremes of age. Most children are infected with M. 
tuberculosis in their home by some one close to them. But they rarely infect other children or adults. ${ }^{1}$

Disseminated or meningeal tuberculosis are most fatal and are early manifestations, often occurring within 2-6 months of the infection. ${ }^{1}$ The most clinically significant form of disseminated tuberculosis is milliary disease, which is most common in infants and young children. Because this form of tuberculosis is most common in infants and in malnourished or immunosuppresed patients, the hostimmune in competency probably also plays a significant role in pathogenesis. ${ }^{1}$

\section{CASE SUMMARY}

Sayeed, 5-months, immunized as per EPI schedule, $3^{\text {rd }}$ sib (out of 3 ) of a nonconsanguinous parents residence at slum area at downtown, Dhaka was admitted on $11^{\text {th }}$ March 2004 with fever for 1 month, dry cough for 21 days, loose motion and vomiting for 14 days. Fever was low grade, occasionally become higher and mostly comes at night with profuse sweating. There was no history of convulsion, unconsciousness or ear discharge. For last 21 days along with fever he developed dry cough that was gradually deteriorating. He also developed loose motion for last 14 days that was very smelly but not bloody associated with vomiting containing food particles about 9-10 times / day. He was treated for several times by several antibiotics without any satisfactory result. Sayeed is borned normally at term in a clinic and cried just after birth. His mother was on regular antenatal care and her pregnancy was uneventful. He had history of early weaning from exclusive breast feeding within first 3 months of his age and replaced by formula as well as cows milk with feeder. He thrived well upto his first 3 months of age. BCG vaccine was given at 6 weeks. There was no history of contact with known case of TB patient although his mother had been suffering from recurrent febrile illnesses and weight loss for many months. Sayeed was looking ill but conscious, mildly pale, afebrile with no dyspnoea and facial dysmorphism. He was mildly dehydrated but not icteric, oedematous or cyanosed. His weight, supine length and OFC were $5.5 \mathrm{~kg}, 59 \mathrm{~cm}$ and $43 \mathrm{~cm}$ respectively. Z score of wt/age, Ith/age, $w t /$ th and OFC were $-2.9,-3.5,-0.3$ and -0.3 respectively. Fontanel was open and normal. Heart rate was $150 /$ min and respiratory rate $32 / \mathrm{min}$. There was no lymphadenopathy and BCG mark is present. Breath sound was vesicular with fine crepitation especially on right lung. Heart sounds were audible in all 4 areas with no murmur. All other systemic enquiry revealed normal. He was treated initially as septicemia by combined parenteral antibiotics covering gram-negative organism. But response was not satisfactory. Lab data yielded raised ESR $(70 \mathrm{~mm})$, eosinophillia (5.0\%), pyuria (50-60/HPF) and haematuria (10-20/HPF) with sterile culture. Chest $X$ ray (A/P and Lateral view) revealed patchy densities along paracardiac as well as parahilar region on right side and also in left parahilar region. The radiological advice was to correlate clinically and laboratory findings to exclude tuberculosis. MT was done and response was much exaggerated with a reading of $18 \mathrm{~mm}$ after $72 \mathrm{hrs}$. All other members of his family were immediately screened for tuberculosis and revealed positive evidenced by MT $(30 \mathrm{~mm}, 28 \mathrm{~mm}$ and $25 \mathrm{~mm}$ after 72 hours found in father, mother and brother respectively) with raised ESR. Regarding chest X-ray, other than his brother (revealed right hilar lymph adenopathy and radiological advice was to exclude tuberculosis) all other family members found nothing significant abnormal findings. Except mother, rest of the family members was asymptomatic. But the patient's father was a smoker and had history of recurrent episodes of dry cough. We started anti tubercular therapy to all of them for 6 months and 12 months for Sayeed. We also corrected the feeding practice of the patient and kept in close monitoring. He showed remarkable improvement as he became playful, appetite increased; frequency of loose motion decreased and gained his weight from $5.5 \mathrm{~kg}$ to $5.7 \mathrm{~kg}$. After 11 days of admission we discharged him as disseminated tuberculosis with having pulmonary tuberculosis among all other family members and advised for follow up. They all completed full course of drugs and became fully cured. At present they are healthy. Sayeed is now 4 years of age and his weight is $15 \mathrm{~kg}$ (Median 16.5kg) and height is $98 \mathrm{~cm}$ (Median 102 cm) with good physical and mental growth. 


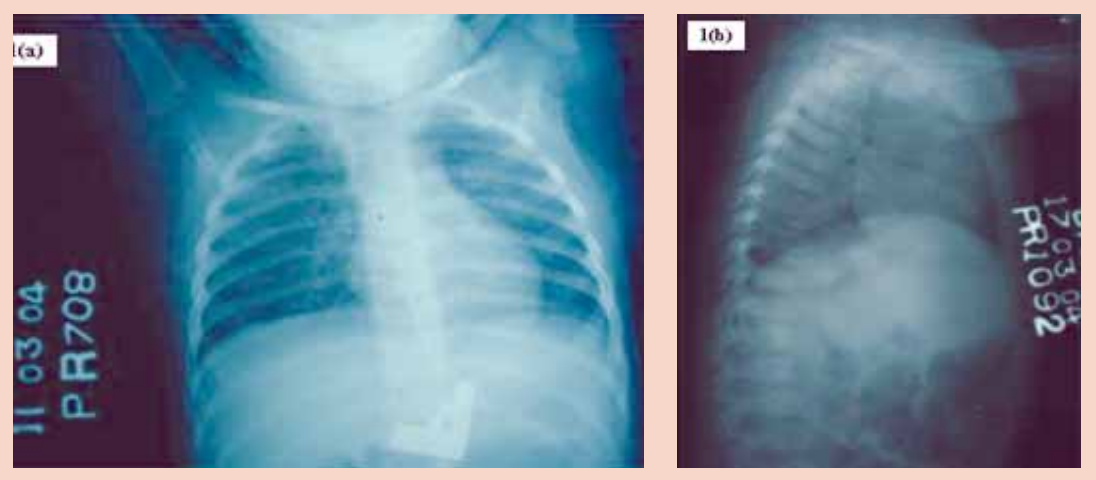

Figure 1(a,b): Chest $X$ ray (A/P and Lateral view) of Sayeed

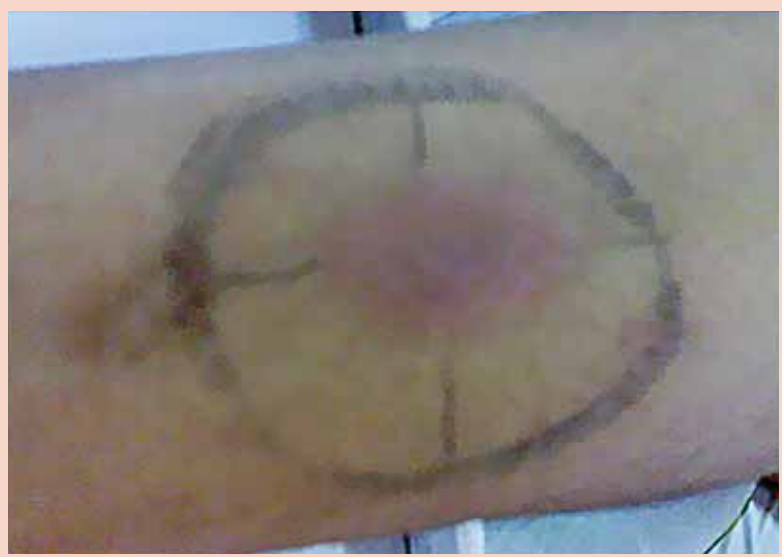

Figure 2: Positive induration of MT
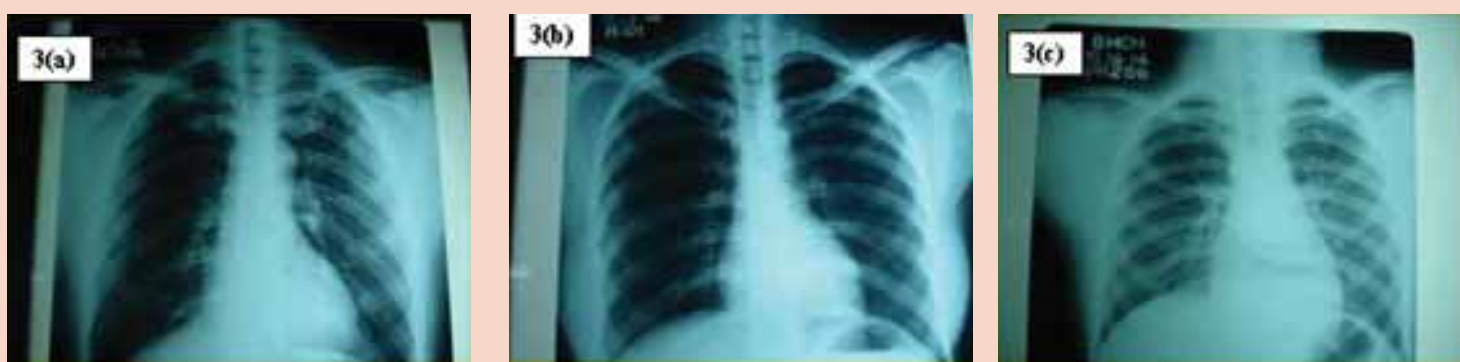

Figure 3: Chest $X$ ray of Father (a), Mother(b) and Brother(c)

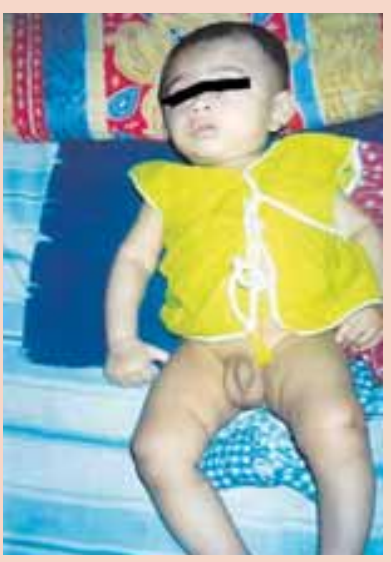

Figure 4: Sayeed at 5 month of age (During admission \& before treatment)

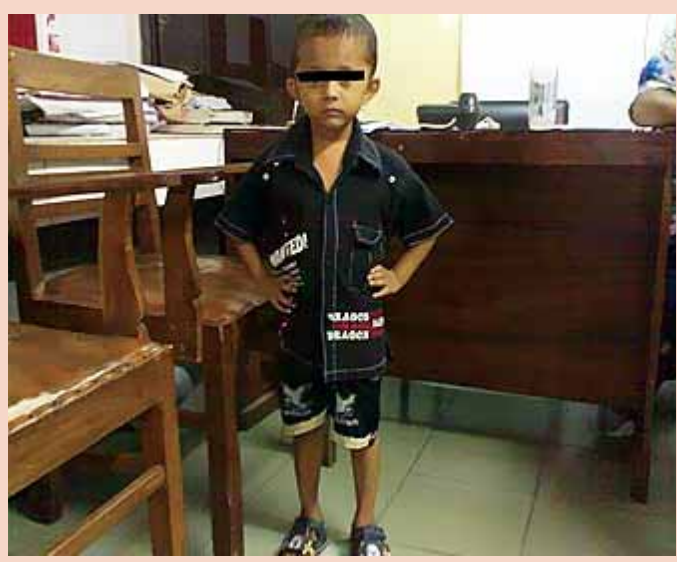

Figure 5: Sayeed with good physique (At present after treatment) 


\section{DISCUSSION :}

The number of tuberculosis among infant is three times larger than those of one or two years old children because of their weak immune systems. ${ }^{5,6}$

Infants who have regular contact (such as living in the same house) with someone who has active (infectious) tuberculosis (TB) have an increased risk of becoming infected. ${ }^{7}$ Our patient had a history of close contact with TB patients as all other of his family members were infected with TB.

Environmental factors also contribute to the likelihood of acquiring the infection. The concentration of bacilli depends on the ventilation of the surroundings and exposure to ultraviolet light. Thus, overcrowding, poor housing, and inadequate ventilation predispose individuals to the development of TB. ${ }^{3}$ These were all present in our case.

The main determinant of the pathogenicity of TB is its ability to escape host defense mechanisms. ${ }^{3,8}$

Most infants with tuberculosis are symptomatic at the time of diagnosis, and bacteriologic confirmation was obtained in $70 \%$ of cases. A study in Texus done between 1985 and 1992 found Seventy-nine percent symptomatic when diagnosed, whereas most of the older children are not. The two most common symptoms were cough and fever. Fever usually low grade and comes at night, although could be as high as $39^{\circ} \mathrm{C}$ and lasted for an average of 14 to 21 days. All fever had resolved in 98 percent of patients by 10 weeks. ${ }^{9,10,11}$ But in another study done in South Africa, 2004 where $69.8 \%$ of cases were found asymptomatic at the time of screening ${ }^{12}$. Our patient was symptomatic at diagnosis and had similar pattern of fever for a month and cough as well. Moreover, our patient was malnourished and early weaned from breast milk (at 3 months); substituted by infant formula and cow's milk in a faulty way caused him more immunosuppressed and made vulnerable to develop TB. These may be the additional important causes to develop weight loss according to age, reluctant to feed and persistent diarrhoea.

Diagnosis of Disseminated TB in infants is difficult enough and a high index of suspicion is required. Bacteriologic confirmation is also very difficult and the tuberculin skin test is frequently negative in patients of this age group with Disseminated TB. The most important clue is history of contact to an adult with infectious tuberculosis ${ }^{13}$. We diagnosed our case from high index of suspicion and by clinical history that was supported by positive tuberculin test as well as abnormal chest X-ray. According to TB Score Chart for use in the Diagnosis of TB in Children Adopted from World Health Organization our patient scored ' 7 ' (as duration of illness more than 4 weeks, nutritional status wt/age within $60 \%-80 \%$ and Positive Mantoux test scored 3,1 and 3 respectively and collectively scored 7) indicates a high likelihood of TB, starting treatment is justified ${ }^{14}$. The presence of acid-fast-bacilli (AFB) on a sputum smear or other specimen often indicates TB disease. Although acidfast microscopy is easy and quick, it does not confirm a diagnosis of TB because some acid-fast-bacilli are not $\mathrm{M}$. tuberculosis. Culture can be done on all initial samples to confirm the diagnosis but a positive culture is not always necessary to begin or continue treatment for $\mathrm{TB}^{15}$. Moreover, the chance of AFB stain of urine is not so high and AFB culture and sensitivity is usually takes 6 weeks and is not available in our institute. As the condition of our patient was so critical and the antibiotic therapy failed, we resorted to antitubercular therapy (ATT) by clinical decision. The child responded promptly. So, we did not go for AFB stain and AFB culture and sensitivity of urine of our patient.

We also screened all other family members to find out any active TB and Complete Blood Count, MT and Chest $X$ ray were chosen as screening methods. We could not do the sputum examination of patient's other family members due to their inconvenience. It may be our drawback but the TB is still considered as social curse by many families in Bangladesh. Parents especially father initially did not show any co-operation with us and even after our proper counseling for doing screening for tuberculosis, he was repeatedly refusing to give consent from doing so not only for himself but also for his spouse. After continuous counseling he at last gave consent for himself and for his wife and other sibs only for tuberculin test, blood count and chest X-ray.

Treatment is very difficult in infants having tuberculosis. One of major reasons is the rapid progress of the disease because of their underdeveloped cell-mediated immunity. Delay in the diagnosis also makes the treatment difficult. During treatment, systemic and enteric infections and liver functional disorders caused by these infections sometimes disturb the treatment for tuberculosis ${ }^{5}$. Fortunately, we did not find any such difficulties during the period of anti tubercular therapy (ATT) in our case. Probably early diagnosis and early initiation of ATT save the infant from facing various treatment related hazards. Moreover, family 
members of our patient who had also TB did not suffer from any treatment induced complications too.

We commenced ATT as a traditional way and not as DOTS. We also corrected the feeding practice of the patient and kept in close monitoring. Although we could not show any bacteriological proof of having tuberculosis of our patient as well as his other family members and treated them with ATT on the basis of high index of suspicion and clinical history that was supported by some laboratory findings, we found satisfactory results evidenced by remarkable clinical improvement of our patient and his other family members as well. Tuberculosis is the disease where bacteriological proof is very difficult especially in children and all the diagnostic criteria cannot be fulfilled properly. If we do not go for ATT to a patient who actually has TB on the basis of high index suspicion due to lack of proof of bacteriological identification, it may be fatal.

\section{CONCLUSION}

TB can mimic any symptom and any sign. A high index of suspicion by the clinician is essential for early diagnosis. The prognosis is excellent if the diagnosis is made early and is often life saving.

\section{MESSAGE}

Family members of affected child must be screened for active TB.

\section{REFERENCES}

1. Starke JR, Munoz FM. Mycobacterial Infections. Tuberculosis (Mycobacterium tuberculosis) In: Behrman RE, Kliegman RM, Jenson HB (eds). Nelson Textbook of Pediatrics, Eighteenth Edition, Saunders, 2007; 1(XVI);7(212): 12401253.

2. WHO Guideline. Treatment of Tuberculosis: Guidelines for National Pragrammes. World Health Organization - Geneva, Third Edition, 2003; 1:11.

3. Internet: Batra V, Ang JY. Article: Tuberculosis. Last Updated: June, 2006; www.eMedicine.com
4. National Guidelines and Operational Manual for Tuberculosis Control. National Tuberculosis Control Program, Directorate General of Health Services, Third Edition, Dhaka, Bangladesh, 2006; 1(1.1):13.

5. Kondo S, Ito M. Difficulties in the treatment of tuberculosis in infants. Kekkaku, 2003; 78(1):1-3.

6. Internet: Feature article: Infant Screening: Tuberculosis. Last Reviewed: March, 2008. @2001-2008 American Association for Clinical Chemistry. Email concerns to Lab Tests Online.

7. Internet: Essig MG., ELS MS. Tuberculosis in infants and children. Last Updated: May, 2007. http://www. revolutionhealth.com/conditions/lung/tuberculosis/diagnose/ children

8. Khan EA, Starke JR. Diagnosis of Tuberculosis in Children: Increased Need for Better Methods. EID 1995;1(4)

9. Vallejo JG, Ong LT, Starke JR. Clinical Features, Diagnosis, and Treatment of Tuberculosis in Infants. PEDIATRICS 1994; 94(1): 1-7.

10. Dershewitz RA. Tuberculosis in Infants. J Watch Gen Med 1994.

11. Internet: Basgoz N. Clinical manifestations of pulmonary tuberculosis. Last Updated: February, 2005. httpwww.uptodateonline.compatientscontenttopic. dotopicKey= bE7160eXis.pyE

12. Kruk A, Gie RP, Schaaf HS, Marais BJ. Sympom - based screening of child tuberculosis contacts: improved feasibility in resource - limited settings. Pediatrics 2008;121(6):e164652.

13. Kim WS, Choi JII, Cheon J-E, Kim I-O, Yeon KM, Lee HJ. Pulmonary Tuberculosis in Infants: Radiographic and CT Findings. AJR 2006; 187:1024-1033.

14. National Guidelines and Operational Manual for Tuberculosis Control. National Tuberculosis Control Program, Directorate General of Health Services, Third Edition, Dhaka, Bangladesh, 2006; $11(\mathrm{~F}): 61$.

15. Internet: Diagnosis of Tuberculosis Disease. TB Elimination Department of Health \& Human Services - USA, Centers for Disease Control and Prevention (CDC), August, 2009: 1-2. http://www.cdc.gov/tb/publications/factsheets/testing/ diagnosis.pdf 